\title{
VIII. Alternating currents in concentric cables
}

\section{W. A. Price M.A.}

To cite this article: W. A. Price M.A. (1897) VIII. Alternating currents in concentric cables, Philosophical Magazine Series 5, 44:266, 61-74, DOI: 10.1080/14786449708621029

To link to this article: http://dx.doi.org/10.1080/14786449708621029

曲 Published online: 08 May 2009.

Submit your article to this journal

Џ Article views: 2

Q View related articles $₫$ 


\section{$\left[\begin{array}{ll}61 & 1\end{array}\right.$}

VIII. Alternating Currents in Concentric Cables. By W. A. Price, M.A.*

Fig. 1.

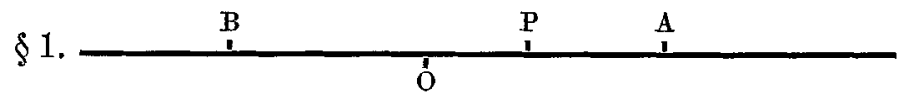

$A O B$ is part of an infinite electrical conductor possessing resistance and capacity, the inductance being negligible. The characteristic differential equation is

$$
\frac{d^{2} u}{d x^{2}}=\rho \gamma \frac{d u}{d t}
$$

$\rho$ being the resistance, and $\gamma$ the capacity per unit of length. At A, B are inserted equal alternating electromotive forces. in the same phase, each represented by $2 \mathrm{E} \sin \omega t$, so that there is no current at $O$ the middle point of $\triangle B$. The distance $A B$ is $2 \mathrm{~L}$. At any point $\mathrm{P}$ distant $x$ from $\mathrm{O}$ the potential due to the electromotive force acting at $A$ is

where

$$
\mathrm{E} e^{-\alpha(\mathrm{L}-x)} \sin \{\omega t-\alpha(\mathrm{L}-x)\}
$$

$$
\alpha=\sqrt{\frac{\omega \rho \gamma}{2}},
$$

and the current at $\mathrm{P}$ due to this force acting at $\mathrm{A}$ is

$$
\frac{\mathrm{E}}{\sqrt{2}} \frac{\alpha}{\rho} e^{-\alpha(\mathrm{L}-x)}[\sin \{\omega t-\alpha(\mathrm{L}-x)\}+\cos \{\omega t-\alpha(\mathrm{L}-x)\}] .
$$

The current at $\mathrm{P}$ due to the electromotive force acting at $B$ is

$$
\frac{\mathrm{E}}{\sqrt{2}} \cdot \frac{\alpha}{\rho} e^{-\alpha(\mathrm{L}+x)}[\sin \{\omega t-a(\mathrm{~L}+x)\}+\cos \{\omega t-\alpha(\mathrm{L}+x)\}]
$$

acting in an opposite direction to the first.

The potential at $\mathrm{P}$ due to the electromotive force acting at $B$ is

$$
E e^{-\alpha(\mathrm{L}+x)} \sin \{\omega t-\alpha(\mathrm{L}+x)\} .
$$

Then the resultant potential at $A$ is

$$
\mathrm{E}\left\{\sin \omega t+e^{-2 \alpha \mathrm{L}} \sin (\omega t-2 \alpha \mathrm{L})\right\} \text {; }
$$

the potential at $\mathrm{O}$ is

$$
2 \mathrm{E} e^{-a \mathrm{I}} \sin (\omega t-\alpha \mathrm{L})
$$

* Communicated by the Physical Society : read April 9, 1897. 
the current at $\mathrm{A}$ is

$$
\frac{\mathrm{E}}{\sqrt{2}} \cdot \frac{\alpha}{\rho}\left\{1-2 e^{-2 \alpha \mathrm{L}} \cos 2 \alpha \mathrm{L}+e^{-4 \alpha \mathrm{L}}\right\}^{\frac{1}{2}} \cos \omega t .
$$

Changing the time from which $t$ is reckoned, so that $\omega t$ becomes $\omega t+\phi$, where

and writing

$$
\tan \phi=\frac{1-e^{-2 \alpha \mathrm{L}}(\cos 2 \alpha \mathrm{L}+\sin 2 \alpha \mathrm{L})}{1-e^{-2 \alpha \mathrm{L}}(\cos 2 \alpha \mathrm{L}-\sin 2 \alpha \mathrm{L})},
$$

$$
\frac{\mathrm{E}}{\sqrt{2}} \cdot \frac{\alpha}{\rho}\left\{1-2 e^{-2 \alpha \mathrm{I}} \cos 2 \alpha \mathrm{L}+e^{-4 \alpha \mathrm{L}}\right\}^{\frac{1}{2}} \text { as } \mathrm{A} \text {, and } \mathrm{L} \gamma \text { as } \Gamma,
$$

the current at $A$ becomes $A \cos \omega t$;

the potential at $\mathrm{A}$ becomes

$\frac{\mathrm{A}}{\omega \Gamma} \alpha \mathrm{L} \frac{(\sinh 2 \alpha \mathrm{L}+\sin 2 \alpha \mathrm{L}) \sin \omega t+(\sinh 2 \alpha \mathrm{L}-\sin 2 \alpha \mathrm{L}) \cos \omega t}{\cosh 2 \alpha \mathrm{L}-\cos 2 \alpha \mathrm{L}}$;

and the potential at $O$ becomes

$\frac{2 \mathrm{~A}}{\omega \Gamma} \alpha \mathrm{L} \frac{(\cos a \mathrm{~L} \sinh a \mathrm{~L}+\sin \alpha \mathrm{L} \cosh a \mathrm{~L}) \sin \omega t+(\cos a \mathrm{~L} \sinh a \mathrm{~L}-\sin a \mathrm{~L} \cosh a \mathrm{~L}) \cos \omega t}{\cosh 2 \alpha \mathrm{L}-\cos 2 a \mathrm{~L}}$.

The form and notation of these expressions are those given in 'Alternating Currents of Electricity,' T. H. Blakesley, London, 1889.

Consider the circular electrical conductor $\mathrm{AB}$ (fig. 2), having a total resistance $2 \pi r$ and a total capacity $2 \pi c$ : the characteristic equation being

$$
\frac{d^{2} u}{d \theta^{2}}=r c \cdot \frac{d u}{d t} \text {. }
$$

At a time $t=0$, when the con-

Fig. 2.

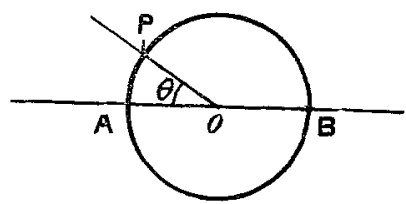
ductor is uncharged, a charge $2 \pi \mathrm{S}$ is introduced at $\mathrm{A}$. The charge at any point $\mathrm{P}$ at a subsequent time $t$ is

$$
s=\mathrm{S} \sum_{-\infty}^{\infty} \cos i \theta e^{-\mu i^{2} t}, \text { where } \mu=\frac{1}{r c}:
$$

$s d \theta$ being the charge at $\mathrm{P}$, where $\mathrm{AOP}=\theta$, of a small arc subtending $d \theta$ at the centre.

If a series of charges, $2 \pi \mathrm{S} \cos \omega \tau . d \tau$, is introduced at $A$ at intervals of time $d \tau$ beginning when $\tau=0, s$, the charge at $P$ at a time $t$,

$$
=\mathbb{S} \sum_{-\infty}^{\infty} \cos i \theta\left\{\frac{\mu i^{2} \cos \omega t+\omega \sin \omega t}{\omega^{2}+\mu^{2} i^{4}}-\frac{\mu i^{2}}{\omega^{2}+\mu^{2} i^{4}} e^{-\mu i^{2} t}\right\} .
$$


If this succession of charges equivalent to a permanent alternating current $2 \pi \mathrm{S} \cos \omega t$ be continued for a long time, the term involving $e^{-\mu i^{2}}$ becomes very small and we have

$$
s=\mathrm{S} \sum_{-\infty}^{\infty} \cos i \theta \frac{\mu i^{2} \cos \omega t+\omega \sin \omega t}{\omega^{2}+\mu^{2} i^{4}} .
$$

The potential at $\mathrm{A}$ is

$$
\frac{\mathrm{S}}{\omega c} \sin \omega t \sum_{-\infty}^{\infty}\left(1+\frac{\mu^{2} i^{4}}{\omega^{2}}\right)^{-1}+\frac{\mathrm{S}}{\omega c} \cos \omega t \sum_{-\infty}^{\infty} \frac{\mu i^{2}}{\omega}\left(1+\frac{\mu^{2} i^{4}}{\omega^{2}}\right)^{-1} ;
$$

the potential at $\mathrm{B}$ is

$$
\frac{\mathrm{S}}{\omega c} \sin \omega t \sum_{-\infty}^{\infty}(-1)^{i}\left(1+\frac{\mu^{2} i^{1}}{\omega^{2}}\right)+\frac{\mathrm{S}}{\omega c} \cos \omega t \sum_{-\infty}^{\infty}(-1)^{i} \frac{\mu i^{2}}{\omega}\left(1+\frac{\mu^{2} i^{4}}{\omega^{2}}\right)^{-1} .
$$

It may be seen that the electrical actions in the two cases discussed are identical so far as the portion $A B$ of the conductor in the first case is concerned.

$\underset{\alpha \mathrm{L}=\beta \pi,}{\text { Writing } \mathrm{L} \gamma=\pi c, \mathrm{~L} \rho=\pi v, \pi \mathrm{S}=\mathrm{A} \text {, and } \sqrt{\frac{\omega}{2 \mu}} \text { as } \beta \text {, so that }}$

$$
\sum_{-\infty}^{\infty}\left(1+\frac{i^{4}}{4 \beta^{4}}\right)^{-1}=\beta \pi \frac{\sinh 2 \beta \pi+\sin 2 \beta \pi}{\cosh 2 \beta \pi-\cos 2 \beta \pi}
$$

[this expression I shall refer to as $\left.f_{1}(\beta)\right]$;

$$
\sum_{-\infty}^{\infty} \frac{i^{2}}{2 \beta^{2}}\left(1+\frac{i^{4}}{4 \beta^{4}}\right)^{-1}=\beta \pi \frac{\sinh 2 \beta \pi-\sin 2 \beta \pi}{\cosh 2 \beta \pi-\cos 2 \beta \pi}
$$

[this expression I shall refer to as $\left.f_{2}(\beta)\right]$;

$$
\sum_{-\infty}^{\infty}(-1)^{i}\left(1+\frac{i^{4}}{4 \beta^{4}}\right)^{-1}=2 \beta \pi \frac{\cos \beta \pi \sinh \beta \pi+\sin \beta \pi \cosh \beta \pi}{\cosh 2 \beta \pi-\cos 2 \beta \pi}
$$

[this expression I shall refer to as $\left.f_{3}(\beta)\right]$;

$$
\sum_{-\infty}^{\infty}(-1)^{i} \frac{i^{2}}{2 \beta^{2}}\left(1+\frac{i^{4}}{4 \beta^{4}}\right)^{-1}=2 \beta \pi \frac{\cos \beta \pi \sinh \beta \pi-\sin \beta \pi \cosh \beta \pi}{\cosh 2 \beta \pi-\cos 2 \beta \pi}
$$

[this expression I shall refer to as $f_{4}(\beta)$ ].

In the particular case where $\beta$ is integral

$$
\begin{aligned}
& \sum_{-\infty}^{\infty}\left(1+\frac{i^{4}}{4 \beta^{4}}\right)^{-1}=\sum_{-\infty}^{\infty} \frac{i^{2}}{2 \beta^{2}}\left(1+\frac{i^{4}}{4 \beta^{4}}\right)^{-1}=\beta \pi \operatorname{coth} \beta \pi, \\
& \sum_{-\infty}^{\infty}(-1)^{i}\left(1+\frac{i^{4}}{4 \beta^{4}}\right)^{-1}=\sum_{-\infty}^{\infty}(-1)^{i} \frac{i^{2}}{2 \beta^{2}}\left(1+\frac{i^{4}}{4 \beta^{4}}\right)^{-1}=\beta \pi \operatorname{cosech} \beta \pi .
\end{aligned}
$$


These last expressions may also be obtained by expanding $(\cos \beta \theta \pm \sin \beta \theta) e^{\beta \theta}$ in Fourier series of cosines.

For

$$
\begin{aligned}
\beta \pi(\cos \beta \theta & -\sin \beta \theta) e^{\beta \theta} \\
& =\sum_{-\infty}^{\infty}(-1)^{i}\left\{(-1)^{\beta} e^{\beta \pi}-(-1)^{i}\right\}\left\{1+\frac{i^{4}}{4 \beta^{4}}\right\}^{-1} \cos i \theta,
\end{aligned}
$$

$\beta \pi(\cos \beta \theta+\sin \beta \theta) e^{\beta \theta}$

$$
\left.=\sum_{-\infty}^{\infty}(-1)^{i}{ }_{1}(-1)^{\beta} e^{\beta \pi}-(-1)^{i}\right\} \frac{i^{2}}{2 \beta^{2}}\left\{1+\frac{i^{4}}{4 \beta^{4}}\right\}^{-1} \cos i \theta .
$$

Putting $\theta$ equal to 0 and $\pi$ successively in these series, and adding and subtracting the series thus obtained in pairs, the four last expressions are obtained. The method is applicable only when $\beta$ is integral.

In a paper by Dr. Glaisher ('Proc. London Math. Soc.,' vol. vii.) the value of $\sum_{-\infty}^{\infty} \frac{1}{r^{4}+x^{4}}$ is determined by another method which gives the same result as the above.

The physical significance of $\beta$ being integral is that in that case the value of $\omega$ is such that the alternations at the sending and receiving ends of the cable are in the same phase. The charge at the sending end being

$$
s=\frac{\mathrm{S}}{\omega} \beta \pi \cosh \beta \pi(\sin \omega t+\cos \omega t)
$$

and at the receiving end

$$
s=\frac{\mathrm{S}}{\omega} \beta \pi \operatorname{cosech} \beta \pi(\sin \omega t+\cos \omega t),
$$

the ratio of the amplitudes beiing $\cosh \beta \pi$.

$\S 2$. A cable, shown in section in fig. 3 , contains two conductors, an inner central conductor and an outer concentric conductor, insulated from one another and the water in which the whole is immersed. The whole cable is supposed to be arranged in the circular form of fig. 2 .

Fig. 3.

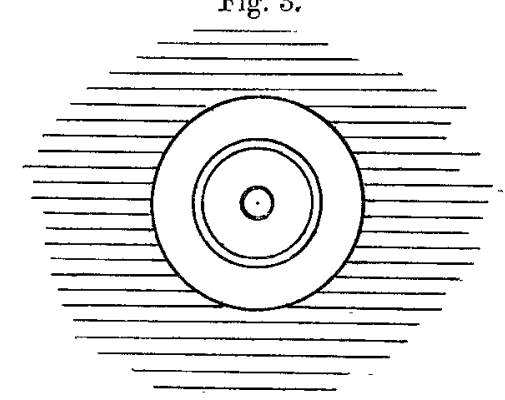

$2 \pi r, 2 \pi r^{\prime}$ are the total resistances of the inner and outer conductors ; 
$2 \pi m$ the total capacity of the inner dielectric measured between the two conductors;

$2 \pi n$ the total capacity of the outer dielectric measured between the outer conductor and the water.

Then $2 \pi \frac{m n}{m+n}$ is the capacity of the central conductor, the outer one being insulated; and $2 \pi n$ the capacity of the outer conductor, the inner one being insulated.

$u u^{\prime}, \mathrm{C}^{\prime} \mathrm{C}^{\prime}$ are the potentials of and the currents in the two conductors at point $\mathrm{P}$ and time $t$, and $s s^{\prime}$ the density of the charges on the conductors. All these quantities being referred to a unit of length subtending unit angle at the centre, then

$$
\begin{gathered}
r \mathrm{C}=-\frac{d u}{d \theta}, \quad r^{\prime}\left(\mathrm{C}^{\prime}=-\frac{d u^{\prime}}{d \theta^{-}} ;\right. \\
-\frac{d \mathrm{C}}{d \theta}=m\left(\frac{d u}{d t}-\frac{d u^{\prime}}{d t}\right), \quad-\frac{d \mathrm{C}^{\prime}}{d \theta}=m\left(\frac{d u^{\prime}}{d t}-\frac{d u}{d t}\right)+n \frac{d u^{\prime}}{d t} ; \\
s+s^{\prime}=n u^{\prime}, \quad \text { and } \quad s=m\left(u-u^{\prime}\right) . \\
\left(\frac{d^{2}}{d \theta^{2}}-m r^{\prime} \frac{d}{d t}\right) s+m r^{\prime} \frac{l}{d t} s^{\prime}=0, \\
\text { and } \quad\left(\frac{d^{2}}{d \theta^{2}}-(m+n) r^{\prime} \frac{d}{d t}\right) s^{\prime}+m r \frac{d}{d t} s=0 .
\end{gathered}
$$

Also

Hence $s$ and $s^{\prime}$ satisfy the equation

$$
\left(\mu \frac{d^{2}}{d \theta^{2}}-\frac{d}{d t}\right)\left(v \frac{d^{2}}{d \theta^{2}}-\frac{d}{d t}\right) s=0,
$$

where $\mu, \nu$ are roots of $m m r^{\prime}: i^{2}-\left(m r^{2}+(m+n) r^{\prime}\right) x+1=0$.

$\S 3$. A uniform conductor $Q$, along which the propagation of charges due to any electrical disturbance is determined by the differential equation

$$
\left(\mu \frac{d^{2}}{d \theta^{2}}-\frac{d}{d t}\right)\left(\nu \frac{d^{2}}{d \theta^{2}}-\frac{d}{d t}\right) s=0,
$$

is arranged in a circle as in fig. 2. Its total resistance is $2 \pi r$, and its total capacity $2 \pi c$. Any disturbance will be propagated along the conductor in two distinct systems, one determined by the differential equation

$$
\left(\mu \frac{d^{2}}{d \theta^{2}}-\frac{d}{d t}\right) s=0, \quad \text { the other by }\left(v \frac{d^{2}}{d \theta^{2}}-\frac{d}{d t}\right) s=0 ;
$$

and the disturbance produced at any given noint will be the sum of two disturbances conveyed by the two systems.

Phil. Mag. S.5. Vol. 44. No. 266. July 1897. F 
Since each propagation is determined by a differential equation of the same form as that which applies to a single isolated conductor, such as is discussed in $\S 1$, the action of $Q$ is equivalent to that of a pair of single conductors, and the propagation along $\mathrm{Q}$ is the sum of the propagations along the equivalent pair.

Let the total resistances of the two equivalent cables be $2 \pi r_{1}$ and $2 \pi r_{2}$, and their capacities $2 \pi c_{1} 2 \pi c_{2}$. Then $\mu r_{1} c_{1}=1$ and $\nu r_{2} c_{2}=1$.

Since the pair is electrically equivalent to $Q$, they must convey the same steady current as $Q$, when subject to a steady E.M.F., and must be charged to the same potential by a given charge. So $r_{1}{ }^{-1}+r_{2}^{-1}=r^{-1}$, and $c_{1}+c_{2}=c$. From these four equations $r_{1} r_{2} c_{1} c_{2}$ can be determined.

Then if a charge $2 \pi \mathrm{S}$ be introduced into this conductor at any point, the charge at any point after a time $t$ will be the sum of the charges at the corresponding points of the equivalent pair, if the same charge had been introduced into them in the proportions $2 \pi \mathrm{S} \frac{c_{1}}{c}, \quad 2 \pi \mathrm{S} \frac{c_{2}}{c}$

If an alternating current $2 \pi \mathrm{S} \cos \omega t$ be introduced into the conductor at $A$ where $\theta=0$, the charge at $\mathbf{P}$ will be

$$
\begin{gathered}
s=\frac{S}{\omega} \frac{c_{1}}{c} \sum_{-\infty}^{\infty} \cos i \theta \frac{\mu i^{2} \cos \omega t+\omega \sin \omega t}{\omega^{2}+\mu^{2} i^{4}} \\
+\frac{\mathrm{S}}{\omega} \frac{c_{2}}{c} \sum_{-\infty}^{\infty} \cos i \theta \frac{\nu^{2} \cos \omega t+\omega \sin \omega t}{\omega^{2}+\nu^{2} i^{4}}=s_{1}+s_{2} .
\end{gathered}
$$

$\$ 4$. Applying this argument to the conductors of the calle described in $\S 2$, let $r_{1} r_{2} c_{1} c_{2}$ be the resistances and capacities of the pair equivalent to the central conductor, and $r_{1}^{2}{ }^{\prime} r_{2}^{\prime} c_{1}^{\prime} c_{2}^{\prime}$ of the pair equivalent to the concentric; so that

$$
\begin{gathered}
r_{1}^{-1}+r_{2}{ }^{-1}=r^{-1}, \quad r_{1}^{\prime}{ }^{\prime}+r_{2}^{\prime-1}=r^{\prime-1} ; \\
r_{1} c_{1} \mu=r_{2} c_{2} \nu=r_{1}^{\prime} c_{1}^{\prime} \mu=r_{2}^{\prime} c_{2}^{\prime} \nu=1 ; \\
c_{1}+c_{2}=\frac{m n}{m+n}=c, \quad c_{1}^{\prime}+c_{2}^{\prime}=c^{\prime}=n .
\end{gathered}
$$

Then

$$
\frac{c_{1}}{c}=\frac{e_{2}^{\prime}}{e^{\prime}}=\frac{\mu}{\mu-\nu}(1-m \nu r),
$$

and

$$
\frac{c_{2}}{c}=\frac{c_{1}^{\prime}}{c^{\prime}}=\frac{\nu}{\nu-\mu}\left(1-m \mu r^{\prime}\right) .
$$

Then the charge at any point $\mathrm{P}$ due to an alternating current $2 \pi \mathrm{S} \cos \omega t$ introduced at $\mathrm{A}$, where $\theta=0$, is $s=s_{1}+s_{2}$, 
where

$$
\begin{aligned}
& s_{1}=\mathrm{S} \frac{\mu}{\mu-\nu}(1-m \mu r) \sum_{-\infty}^{\infty} \cos i \theta \frac{\mu i^{2} \cos \omega t+\omega \sin \omega t}{\omega^{2}+\mu^{2} i^{4}} \\
& s_{9}=\mathrm{S} \frac{\nu}{\nu-\mu}(1-m \nu r) \sum_{-\infty}^{\infty} \cos i \theta \frac{\nu i^{2} \cos \omega t+\omega \sin \omega t}{\omega^{2}+\nu^{2} i^{4}}
\end{aligned}
$$

$\S 5$. If an alternating current $2 \pi \mathrm{S} \cos \omega t$ be introduced into the concentric conductor at $\mathrm{A}$, the charge $s^{\prime}$ at any point $\mathrm{P}=s_{1}{ }^{\prime}+s_{2}{ }^{\prime}$, where

$$
\begin{aligned}
& s_{1}^{\prime}=\frac{\mathrm{S}}{\omega} \frac{\nu(1-\mu m r)}{\nu-\mu} \sum_{-\infty}^{\infty} \cos i \theta \frac{\mu i^{2} \cos \omega t+\omega \sin \omega t}{\omega^{2}+\mu^{2} i^{4}}, \\
& s_{2}^{\prime}=\frac{\mathrm{S}}{\omega} \frac{\mu\left(1-\nu m \nu^{\prime}\right)}{\mu-\nu} \sum_{-\infty}^{\infty} \cos i \theta^{\nu i^{2} \cos \omega t+\omega \sin \omega t}
\end{aligned}
$$

$\$ 6$. Consider the charge $s^{\prime}$ induced in the concentric conductor by the charge $s_{1}+s_{2}$ in the central conductor.

From $\S 2$,

$$
\begin{aligned}
& \left(\frac{d^{2}}{d \bar{\theta}^{\overline{2}}}-m r \frac{d}{d t}\right)\left(s_{1}+s_{2}\right)+m r^{\prime} \frac{d}{d t} s^{\prime}=0 \\
& \frac{d^{2}}{d \theta^{2}} s_{1}=\frac{1}{\mu} \frac{d}{d t} s_{1}, \quad \frac{d^{2}}{d \theta^{2}} s_{2}=\frac{1}{\nu} \frac{d}{d t} s_{2} ;
\end{aligned}
$$

hence

$$
\frac{1-m \mu r}{\mu} \frac{d s_{1}}{d t}+\frac{1-m \nu r}{\nu} \frac{d s_{2}}{d t}+m r^{\prime} \frac{d s^{\prime}}{d t}=0 \text {. }
$$

Integrating, $m r^{\prime} s^{\prime}=-\frac{1}{\mu}(1-m \mu r) s_{1}-\frac{1}{\nu}(1-m v r) s_{2}+$ some expression $\Theta$ independent of $t$.

$\Theta$ is periodic in $\theta$, satisfies $\left(\mu \frac{d^{2}}{d \theta^{2}}-\frac{d}{d t}\right)\left(\nu \frac{d^{2}}{d \theta^{2}}-\frac{d}{d t}\right) \Theta=0$, and has a mean value zero. Hence $\Theta=0$, and

$$
m r^{\prime} s^{\prime}=-\frac{1}{\mu}(1-m \mu r) s_{1}-\frac{1}{\nu}(1-m v r) s_{2} .
$$

In the same way the charge $s$ induced in the central conductor by the distribution $s_{1}^{\prime}+s_{2}^{\prime}$ in the concentric conductor is given by the equations

$$
\begin{aligned}
& \left\{\frac{d^{2}}{d \theta^{2}}-(m+n) r^{\prime} \frac{d}{d t}\right\}\left(s_{1}^{\prime}+s_{2}{ }^{\prime}\right)+m r^{2} \frac{d}{d t} s=0, \\
& \frac{d^{2}}{d \theta^{2}} s_{1}^{\prime}=\frac{1}{\mu} \frac{d}{d t} s_{1}^{\prime}, \quad \frac{d^{2}}{d \theta^{2}} s_{2}^{\prime}=\frac{1}{\nu} \frac{d}{d t} s_{2}^{\prime}, \\
& m r s=\frac{1}{\nu}(1-m r v) s_{1}{ }^{\prime}+\frac{1}{\mu}(1-m r \mu) s_{2}{ }^{\prime} .
\end{aligned}
$$


$\S 7$. Suppose an alternating current $2 \pi A \cos \omega t$ to be introduced into the central conductor at $\mathrm{A}$ where $\theta=0$, and a current $2 \pi \mathrm{B} \cos (\omega t+\phi)$ into the same conductor at $\mathrm{B}$ where $\theta=\pi$.

The charge at $\mathrm{B}$ on the central conductor due to the current introduced at $A$ is, writing

$$
\begin{gathered}
\sqrt{\frac{\omega}{2 \mu}} \text { as } \beta, \text { and } \sqrt{\frac{\omega}{2 \nu}} \text { as } \gamma, \\
\frac{A}{\omega} \frac{c_{1}}{c}\left\{\sin \omega t f_{3}(\beta)+\cos \omega t f_{4}(\beta)\right\} \\
+\frac{A}{\omega} \frac{c_{2}}{c}\left\{\sin \omega t f_{3}(\gamma)+\cos \omega t f_{4}(\gamma)\right\} .
\end{gathered}
$$

The charge at $B$ on the central conductor due to the current introduced at $\mathrm{B}$ is

$$
\begin{aligned}
& \frac{\mathrm{B}}{\omega} \frac{c_{1}}{c}\{\left.\sin (\omega t+\phi) f_{1}(\beta)+\cos (\omega t+\phi) f_{2}(\beta)\right\} \\
&+\frac{\mathrm{B}}{\omega} \frac{c_{2}}{c}\left\{\sin (\omega t+\phi) f_{1}(\gamma)+\cos (\omega t+\phi) f_{2}(\gamma)\right\}
\end{aligned}
$$

The charge at $B$ induced on the concentric conductor by the current introduced at $\mathrm{A}$ is

$$
\begin{aligned}
& -\frac{1}{m \mu r^{\prime}}(1-m \mu r) \frac{\mathrm{A}}{\omega} \frac{c_{1}}{c}\left\{\sin \omega t f_{3}(\beta)+\cos \omega t f_{4}(\beta)\right\} \\
& -\frac{1}{m \nu r^{\prime}}(1-m v \gamma) \frac{\mathrm{A}}{\omega} \frac{c_{2}}{c}\left\{\sin \omega t f_{3}(\gamma)+\cos \omega t f_{4}(\gamma)\right\} .
\end{aligned}
$$

The charge at $B$ induced on the concentric conductor by the current introduced at $B$ is

$$
\begin{aligned}
&- \frac{1}{m \mu r^{\prime}}(1-m \mu r) \frac{\mathrm{B}}{\omega} \frac{c_{1}}{c}\left\{\sin (\omega t+\phi) f_{1}(\beta)+\cos (\omega t+\phi) f_{2}(\beta)\right\} \\
&-\frac{1}{m \nu r^{\prime}}(1-m \nu r) \frac{\mathrm{B}}{\omega} \frac{c_{2}}{c}\left\{\sin (\omega t+\phi) f_{1}(\gamma)+\cos (\omega t+\phi) f_{2}(\gamma)\right\}
\end{aligned}
$$

The potential of the central conductor at the point $B$ is $\frac{(m+n) s+m s^{\prime}}{m n}$, where $s s^{\prime}$ are the total charges on the two conductors respectively, and is 


$$
\begin{gathered}
\operatorname{\mu r} \frac{\mathrm{A}}{\omega} \frac{c_{1}}{c}\left\{\sin \omega t f_{3}(\beta)+\cos \omega t f_{4}(\beta)\right\} \\
+\nu r \frac{\mathrm{A}}{\omega} \frac{c_{2}}{c}\left\{\sin \omega t f_{3}(\gamma)+\cos \omega t f_{4}(\gamma)\right\} \\
+\mu r \frac{\mathrm{B}}{\omega} \frac{c_{1}}{c}\left\{\sin (\omega t+\phi) f_{1}(\beta)+\cos (\omega t+\phi) f_{2}(\beta)\right\} \\
+\nu r \frac{\mathrm{B}}{\omega} \frac{c_{2}}{c}\left\{\sin (\omega t+\phi) f_{1}(\gamma)+\cos (\omega t+\phi) f_{2}(\gamma)\right\} .
\end{gathered}
$$

If a known current $2 \pi \mathrm{A} \cos \omega t$ be introduced at $\mathrm{A}$, and the point $B$ be kept at zero potential, the equation of the last expression to zero for every value of $t$ gives two equations to determine $B$ and $\phi$, the amplitude and phase of the current at the receiving end of the cable. These are

$$
\begin{aligned}
\mathrm{A}\left\{\mu c_{1} f_{3}(\beta)+\nu c_{2} f_{3}(\gamma)\right\} & +\mathrm{B}\left\lfloor\mu c_{1}\left\{\cos \phi f_{1}(\beta)-\sin \phi f_{2}(\beta)\right\}\right. \\
& \left.+\nu c_{2}\left\{\cos \phi f_{1}(\gamma)-\sin \phi f_{2}(\gamma)\right\}\right]=0 ; \\
\mathrm{A}\left\{\mu c_{1} f_{4}(\beta)+\nu c_{2} f_{4}(\gamma)\right\} & +\mathrm{B}\left[\mu c_{1}\left\{\sin \phi f_{1}(\beta)+\cos \phi f_{2}(\beta)\right\}\right. \\
& \left.+\nu c_{2}\left\{\sin \phi f_{1}(\gamma)+\cos \phi f_{2}(\gamma)\right\}\right]=0 .
\end{aligned}
$$

Similarly, if the currents had been introduced into the concentric conductor, the central one being insulated throughout, we should have for the potential of the concentric conductor at $\mathrm{B}, \frac{1}{n}\left(s+s^{\prime}\right)$, which is exactly the same expression as for the central conductor substituting $r^{\prime}$ for $r, \frac{c_{1}{ }^{\prime}}{c}$ for $\frac{c_{1}}{c}$, and $\frac{c_{2}^{\prime}}{c}$ for $\frac{c_{2}}{c}$. Remembering that $\frac{c_{1}}{c}=\frac{c_{2}^{\prime}}{c^{\prime}}$ and $\frac{c_{2}}{c}=\frac{e_{1}^{\prime}}{c^{\prime}}$, it is seen that the equations for determining the phase and amplitude of the current received at $B$ when that end of the concentric conductor is earthed are the same as in the case of the central conductor, only interchanging $c_{1}$ and $c_{2}$.

$\S 8$. So if an alternating current, $2 \pi \mathrm{A} \cos (\omega t+a)$, be introduced into the central conductor at $A$, and a current $2 \pi \mathrm{B} \cos (\omega t+b)$ pass out of the concentric conductor at $\mathrm{B}$, the potentials of the conductors at A, B may be determined.

The potential of the central conductor at $A$ is

$$
\frac{1}{m n}\left\{(n+n) s+m s^{\prime}\right\}
$$

where $s s^{\prime}$ are the charges on the two conductors. 


$$
\begin{aligned}
\text { This } & =\frac{\mathrm{A}}{\omega} \cdot \frac{\mu^{2} r}{\mu-\nu}(1-m v r)\left\{\sin (\omega t+a) f_{1}(\beta)+\cos (\omega t+a) f_{2}(\beta)\right\} \\
& +\frac{\mathrm{A}}{\omega} \cdot \frac{\nu^{2} r^{2}}{\nu-\mu}(1-m \mu r)\left\{\sin (\omega t+a) f_{1}(\gamma)+\cos (\omega t+a) f_{2}(\gamma)\right\} \\
& -\frac{\mathrm{B}}{\omega} \cdot \frac{\mu}{n(\mu-\nu)}\left\{\sin (\omega t+b) f_{3}(\beta)+\cos (\omega t+b) f_{4}(\beta)\right\} \\
& -\frac{\mathrm{B}}{\omega} \cdot \frac{\nu}{(\nu-\mu) n} \cdot\left\{\sin (\omega t+b) f_{3}(\gamma)+\cos (\omega t+b) f_{4}(\gamma)\right\} .
\end{aligned}
$$

The potential of the concentric conductor at B is $\frac{1}{n}\left(s+s^{\prime}\right)$.

$$
\begin{aligned}
\text { This } & =\frac{\mathrm{A}}{\omega} \cdot \frac{\mu}{n(\mu-\nu)}\left\{\sin (\omega t+a) f_{3}(\beta)+\cos (\omega t+a) f_{4}(\beta)\right\} \\
& +\frac{\mathrm{A}}{\omega} \cdot \frac{\nu}{n(\nu-\mu)}\left\{\sin (\omega t+a) f_{3}(\gamma)+\cos (\omega t+a) f_{4}(\gamma)\right\} \\
& +\frac{\mathrm{B}}{\omega} \cdot \frac{\mu \nu r^{\prime}}{\mu-\nu}(1-m \mu r)\left\{\sin (\omega t+b) f_{1}(\beta)+\cos (\omega t+b) f_{2}(\beta)\right\} \\
& +\frac{\mathrm{B}}{\omega} \cdot \frac{\mu \nu r^{\prime}}{\nu-\mu}(1-m \nu r)\left\{\sin (\omega t+b) f_{1}(\gamma)+\cos (\omega t+b) f_{2}(\gamma)\right\} .
\end{aligned}
$$

\$ 9. Consider a system of circular conductors, of which fifteen are shown in fig. 4.

Of these Nos. $1,5,6,10,11,15$ have each a resistance $2 \pi r$; they represent the central conductors of such concentric cables as we have been considering. Nos. $2,4,7,9,12,14$ have each a resistance $2 \pi r^{r}$. They represent the outer or concentric conductors. Nos. 3, 8, 13, have no resistance, and are maintained always at zero potential throughout. They represent the water or sheathing of the cables.

The capacity measured between each of the pairs 1,2 : $4,5: 6,7: 9,10: 11,12: 14,15$ respectively is $2 \pi c$, where $c=m$; and between the pairs $2,3: 3,4: 7,8: 8,9: 12,13$ : 13,14 is $2 \pi c^{\prime}$, where $e^{\prime}=n$. The capacity between the pairs $5,6: 10,11$ is zero.

At $B$ conductors 2, 4 are connected by a piece having no resistance or capacity.

Similarly at C, conductors 5,6 are connected; at D, 7,9; at $\mathrm{E}, 10,11$; at $\mathrm{F}, 12,14$, and so on. The series may be indefinitely extended. 
The system is electrically equivalent to a length of concentric cable of which the two conductors are cut alternately

Fig. 4.

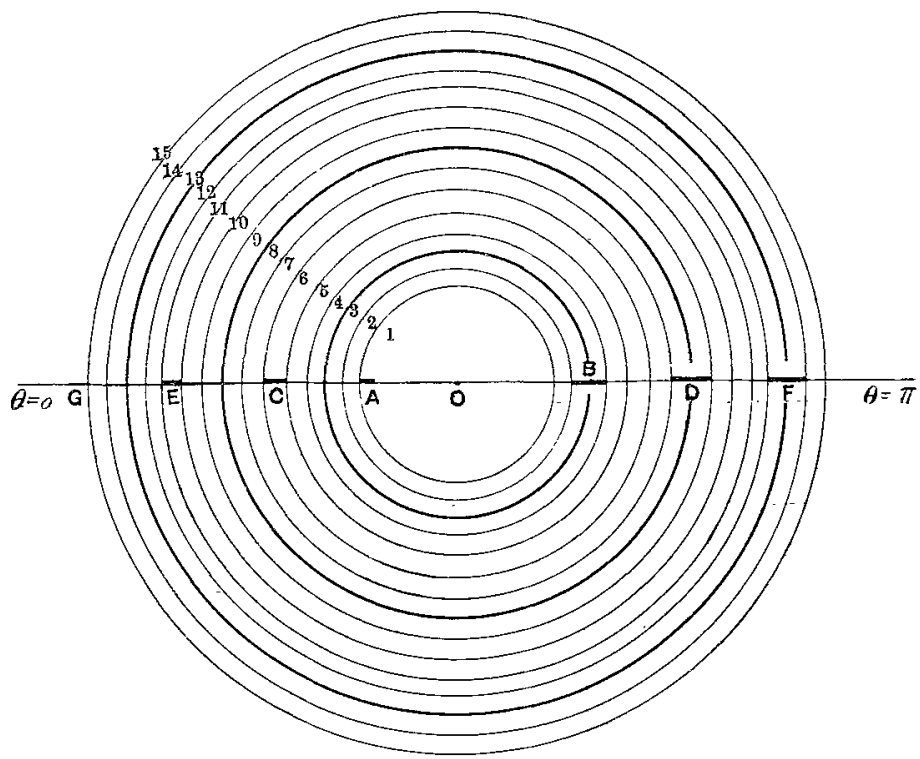

at equidistant points in the way shown in fig. 5, representing a longitudinal section of the cable. If an alternating current be introduced into the system of fig. 4 at any link, say E, and the point A be kept at zero potential, alternating currents of the same period, but of different phases and amplitudes, will be found at every point of both conductors.

Fig. 5.

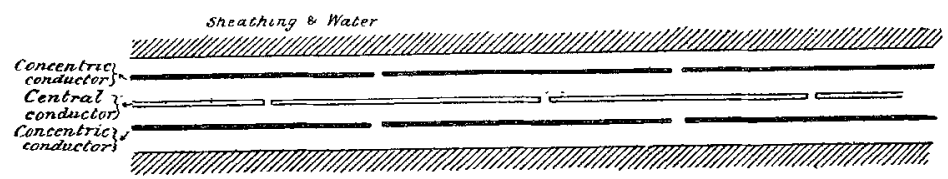

Suppose the currents at the points A, B, C, D, . G to be

$$
\mathrm{A} \cos (\omega t+a), \mathrm{B} \cos (\omega t+b), \ldots \mathrm{G}(\cos \omega t+g),
$$

so that the values of $\mathrm{Gg}$ being known, and the point $\mathrm{A}$ being kept at zero potential, the values of $\mathrm{A} a, \mathrm{~B} b, \mathrm{C} c, \ldots \mathrm{F} f$ are to be determined, and especially the values of $\mathrm{A} a$. 
The potential at the point $\mathrm{C}$ on the conductor 5 has been expressed in terms of $\mathrm{C} c, \mathrm{~B} b$; and the potential at the point $\mathrm{C}$ on the conductor 6 in terms of $\mathrm{C} c, \mathrm{D} d$. These two are equal for all values of $t$, and we have an equation which can be separated into two others between $\mathrm{B} b, \mathrm{C} c, \mathrm{D} d$. In the same way we obtain two equations at $A, B, D, E$, and $F$, and between these all the quantities required can be obtained.

In the particular case where $\omega$ has such a value that $\beta$ and $\gamma$ are integral, and the currents at all the points are in the same phase, let us write in $\S 8$,

$$
\begin{aligned}
& \frac{\mu^{2} r}{\mu-\nu}(1-m \nu r) f_{1}(\beta)+\frac{\nu^{2} r}{\nu-\mu}\left(1-m \mu r^{\prime}\right) f_{1}(\gamma) \text { as } \mathrm{P}, \\
& \frac{\mu}{n(\mu-\nu)} f_{3}(\beta)+\frac{\nu}{n(\nu-\mu)} f_{3}^{\prime}(\gamma) \text { as } \mathrm{Q}, \\
& \frac{\mu \nu r^{\prime}}{\nu-\mu}(1-m \mu r) f_{1}(\beta)+\frac{\mu \nu r^{\prime}}{\mu-\nu}\left(1-m \nu r^{\prime}\right) f_{1}(\gamma) \text { as } \mathrm{R},
\end{aligned}
$$

remembering that when $\beta \gamma$ are integral, $f_{1}(\beta)=f_{2}(\beta)$, and $f_{3}(\beta)=f_{4}(\beta)$, and the same with $\gamma$.

The condition at $A$ gives $A P=B Q$,

$$
\begin{array}{lllll}
" & \Rightarrow & \mathrm{B} & , \quad(\mathrm{A}+\mathrm{C}) \mathrm{Q}=2 \mathrm{BR}, \\
" & \Rightarrow & \mathrm{C} & \Rightarrow & (\mathrm{B}+\mathrm{D}) \mathrm{Q}=2 \mathrm{OP}, \\
& \Rightarrow & \mathrm{D} & \Rightarrow & (\mathrm{C}+\mathrm{E}) \mathrm{Q}=2 \mathrm{DR}, \text { and so on. }
\end{array}
$$

If the known current is introduced at $B$, and $A$ is kept to earth, the ratio of the amplitude of the received current to the sent current is given by

$$
\frac{\mathrm{B}}{\mathrm{A}}=\frac{\mathrm{P}}{\mathrm{Q}} \cdot \cdot \cdot \cdot \cdot \text { C Case I. }
$$

If the known current is introduced at $\mathrm{C}$, we have

$$
\frac{\mathrm{C}}{\mathrm{A}}=2 \frac{\mathrm{PR}}{\mathrm{Q}^{2}}-1 \quad \text {. . . C Case II. }
$$

If the known current is introduced at $\mathrm{E}$, we have

$$
\frac{\mathrm{E}}{\mathrm{A}}=8\left(\frac{\mathrm{PR}}{\mathrm{Q}^{2}}\right)^{2}-8 \frac{\mathrm{PR}}{\mathrm{Q}^{2}}+1 \text {. . Case III. }
$$

If the known current is introduced at $G$, we have

$$
\frac{\mathrm{G}}{\mathrm{A}}=32\left(\frac{\mathrm{PR}}{\mathrm{Q}^{2}}\right)^{3}-48\left(\frac{\mathrm{PR}}{\mathrm{Q}^{2}}\right)^{2}+18 \frac{\mathrm{PR}}{\mathrm{Q}^{2}}-1 . \text { Case IV. }
$$


$\$ 10$. Applying these results to a cable having the following constants, represented by half the circle of fig. 4:

I ength 4000 kilometres $=4.10^{8}$ units.

Resistance of each conductor, 5 ohms per kilometre, i.e. $2.10^{13}$ units for the whole cable.

Capacity measured between the conductors, $\frac{5+\sqrt{7}}{18} 10^{-15}$ units per kilometre (about 425 mfds.), i.e. $\begin{gathered}5+\sqrt{7} \\ 18^{--} \\ 4.10^{-12}\end{gathered}$ units for the whole cable.

Capacity measured between the outer conductor and the sheathing, $\frac{5-\sqrt{7}}{9} 10^{-15}$ units per kilometre (about 26 mfds.), i. e. $\frac{5-\sqrt{7}}{9} \cdot 4 \cdot 10^{-12}$ units for the whole cable.

Then

$$
\begin{gathered}
m=\frac{2}{\pi} \frac{5+\sqrt{7}}{9} 10^{-12}, \quad n=\frac{4}{\pi} \frac{5-\sqrt{7}}{9} 10^{-12}, \\
r=r^{\prime}=\frac{2}{\pi} 10^{13}, \quad \mu=\frac{\pi^{2}}{80}, \quad \nu=\frac{9 \pi^{2}}{80}, \\
\frac{c_{1}}{c}=\frac{c_{2}^{\prime}}{c^{\prime}}=\frac{3+\sqrt{7}}{16}, \quad \frac{c_{2}}{c}=\frac{c_{1}^{\prime}}{c^{\prime}}=\frac{13-\sqrt{7}}{16} .
\end{gathered}
$$

Consider the cable in four cases :-

Case I. when both conductors are continuous.

Case II. when the inner conductor is cut in the middle so that the cable is in two sections.

Case III. when the inner conductor is cut in two places, and the outer in one, so that the cable is in four sections.

Case IV. when the inner conductor is cut in three places, and the outer in two, so that the cable is in six sections.

Take a value of $\omega \cdot \frac{9 \cdot 144 \cdot \pi^{2}}{80}$ which makes $\beta \cdot \gamma$ integral in all cases. This corresponds to about 25 alternations per second.

Then if the cable be divided into $n$ equal sections the new values of $r r^{\prime} m n \beta \gamma$ are inversely proportional to $n$, while the values of $\mu, \nu$ are proportional to the square of $n$. The value of $\frac{P R}{Q^{2}}$ is unchanged in every case. 
In Case I. $\beta=30 \gamma=12$, and $A=1 \cdot 26 e^{-12 \pi} \mathrm{B}$;

Case II. $\beta=18 \gamma=6$, and $\mathrm{A}=1.07 e^{-12 \pi} \mathrm{C}$;

Case III. $\beta=9 \gamma=3$, and $A=.57 e^{-12 \pi} \mathrm{E}$;

Case IV. $\beta=6 \gamma=2$, and $A=\cdot 31 e^{-12 \pi} G$.

Thus the effect of each successive subdivision is to further reduce the amplitude of the received current. viz. :-

\$11. Returning to the equation of $\$ 2$ for determining $\mu \nu$,

write in this

$$
\left.m n v^{\prime} x^{\prime} x^{2}-x \overline{\left\{m+n \nu^{\prime}\right.}+m \nu\right\}+1=0,
$$

$$
\frac{1}{r}=\gamma, \frac{1}{\gamma^{\prime}}=\gamma^{\prime}, \frac{1}{m}=k, \frac{1}{n}=k^{\prime} \text {, and } \gamma+\gamma^{\prime}=\Gamma,
$$

so that $\gamma, \gamma^{\prime}$ are the conductivities of the two conductors, $k, k^{\prime}$ the rigidities to electrostatic stress of the two dielectrics, and $\Gamma$ the total conductivity of the two conductors.

The equation becomes

$$
x^{2}-x\left\{\gamma k+\Gamma k^{\prime}\right\}+\gamma \gamma^{\prime} k k^{\prime}=0,
$$

and the roots of this equation represent the signalling speeds of the two equivalent cables.

If $k$ be very small, $x=\Gamma k^{\prime}$ or 0 . Hence if the layer of dielectric between the conductors be very thin, though at the same time perfectly insulating, the speed through the central conductor, however small its section may be, is the same as if the whole of the two conductors were solid, and the whole used for the conducting circuit.

IX. The Formation of Mercury Filins by an Electrical Process. By Rolito Applemard* *

$\mathrm{I}^{\mathrm{r}}$ F a sheet of gelatine, damp leather, or similar permeable substance is used as a separating diaphragm between two bodies of mercury, and a current is sent through it, a film of mercury is deposited upon the surface connected to the positive pole of the battery. And the film remains on the diaphragm after removal from the apparatus.

If the diaphragm is replaced in the apparatus and subjected to a current in the reverse direction, the film vanishes from that surface, and a second film appears on the other side. That is to say, the film is always on the side of the diaphragm connected to the positive pole of the battery, and there is no film on the negative surface.

* Communicated by the Physical Society : read May 14, 1897. 